\title{
Bone conducted vibration is an effective stimulus for otolith testing in cochlear implant patients
}

\author{
L. Fröhlich ${ }^{1, *}$, M. Wilke ${ }^{1}$, S.K. Plontke and T. Rahne \\ Department of Otorhinolaryngology, Head \& Neck Surgery, Martin Luther University Halle-Wittenberg, \\ Halle (Saale), Germany
}

Received 2 February 2021

Accepted 1 July 2021

\begin{abstract}
.
BACKGROUND: Treatment with a cochlear implant (CI) poses the risk of inducing a behaviorally unmeasurable air-bone gap leading to false negative absence of cervical and ocular vestibular evoked myogenic potentials (cVEMPs, oVEMPs) to air conducted sound (ACS).

OBJECTIVE: To investigate VEMP response rates to ACS and bone conducted vibration (BCV) in CI patients and the applicability of the B81 transducer for BCV stimulation.

METHODS: Prospective experimental study including unilateral CI patients, measuring cVEMPs and oVEMPs to ACS and to $\mathrm{BCV}$, comparing response rates, signed asymmetry ratios, latencies, and amplitudes.

RESULTS: Data of $13 \mathrm{CI}$ patients (mean age $44 \pm 12$ years) were analyzed. For the CI side, oVEMP and cVEMP response rates were significantly higher for BCV (77\% cVEMP, 62\% oVEMP) compared to ACS ( $23 \%$ cVEMP, $8 \%$ oVEMP). For the contralateral side, no difference between response rates to ACS (85\% cVEMP, 69\% oVEMP) and BCV (85\% cVEMP, 77\% oVEMP) was observed. Substantially higher asymmetries were observed for ACS ( $-88 \pm 23 \%$ for cVEMPs, $-96 \pm 11 \%$ for oVEMPs) compared to BCV ( $-12 \pm 45 \%$ for cVEMPs, $4 \pm 74 \%$ for oVEMPs).
\end{abstract}

CONCLUSIONS: BCV is an effective stimulus for VEMP testing in CI patients. The B81 is a feasible stimulator.

Keywords: Cochlear implant, vestibular function, VEMP, air conduction, bone conduction, transducer, air-bone gap, asymmetry

\section{Introduction}

The recording of vestibular evoked myogenic potentials (VEMPs) has become a routine clinical test for the assessment of the otolith organs in the peripheral vestibular system. The recording of VEMPs from the ipsilateral steronocleidomastoid muscle (SCM; cervical, cVEMPs) was first described by Colebatch

\footnotetext{
${ }^{1}$ The authors contributed equally to this work.

*Corresponding authors. Laura Fröhlich, University Hospital Halle, Department of Otorhinolaryngology, Head \& Neck Surgery, Ernst-Grube-Straße 40, 06120 Halle (Saale), Germany. Tel.: +49 345557 1073; E-mail: laura.froehlich@uk-halle.de.
}

et al. [5]. Later, VEMP recordings from contralateral extraocular muscles were reported (ocular, oVEMPs) $[20,37,42]$. Air conducted sound (ACS) with large sound pressure level is used as a common stimulus for activating the otoliths. Recordings in squirrel monkeys showed that saccular afferents had the lowest phase locking threshold to sound [46]. The threshold of saccular afferents to ACS is approximately 15 $20 \mathrm{~dB}$ lower than for utricular afferents. Later it was found that bone conducted vibration (BCV) leading to a series of head accelerations is also a very effective VEMP stimulus. The thresholds of both utricular and saccular afferents are lower to BCV compared to ACS with reference to hearing levels [7, 9]. Differentiation 
between saccular and utricular function is clinically possible due to differences in neural projections. The saccule has an ipsilateral inhibitory projection and the utricle has an ipsilateral inhibitory as well as a contralateral excitatory projection to the SCM [24]. Projections to the inferior oblique muscle are more complex but predominantly of utricular origin. Due to the differences in neural projections and differences in thresholds, ACS is a common stimulus used for cVEMP measurements [9], evaluating saccular function. Utricular function can be assessed by recording the excitatory potentials from the contralateral ocular muscles (oVEMPs). For BCV, activating both saccular and utricular afferents at low stimulation levels, the differentiation is not as clear but cVEMPs indicate predominantly saccular function and oVEMPs indicate predominantly utricular function [8].

While VEMP measurements with ACS are also applicable in patients with profound sensorineural hearing loss, they cannot be elicited in patients with even mild conductive hearing loss. The impairment of sound transmission through the middle and/or inner ear causes reduced VEMP amplitudes or absent responses $[39,48]$. As an alternative, $\mathrm{BCV}$ is a feasible otolithic stimulus for cVEMP as well as oVEMP testing in these patients.

Cochlear implantation is also attributed to causing air-bone gaps [4, 27, 34]. The possible underlying mechanisms can be changes in middle ear but also inner ear mechanics. Changes in middle ear mechanics include fibrous tissue formation after surgery, reaction to the foreign body, and disruption of the ossicular chain [34]. However, Mattingly et al. [27] have shown that the incidence of postoperative airbone gaps did not correlate to abnormalities in tympanometry. Alterations of inner ear mechanics are therefore likely to causing air-bone gaps in cochlear implant (CI) patients. Air-bone gaps of inner ear origin are known from other inner ear pathologies such as large vestibular aqueducts, or semicircular canal dehiscence $[16,29]$. In CI patients, alterations of inner ear mechanics leading to air-bone gaps can be the presence of the electrode as a foreign body itself, inflammatory reactions to the electrode or damage to intracochlear structures leading to fibrosis, and altering compliance of the round window and therefore stapes motion [3, 17, 23, 32]. Also, changes in volume regulation, i.e., endolymphatic hydrops due to the surgical procedure and/or the cochlear implant (CI) array, can occur and affect inner ear mechanics $[15,40]$. In patients with traditional CI indication, i.e., profound sensorineural hearing loss, the air-bone gap following cochlear implantation cannot be measured behaviorally by standard audiometric tests due to the limited maximum output of BC transducers, especially at the low frequencies. Another problem at the low frequencies is the interference with tactile thresholds which makes the interpretation of hearing test results difficult [11]. However, VEMP testing in CI patients is often desirable. Due to the close anatomical proximity of the cochlea and the otolith organs in the vestibule, they are suggested to be at risk with cochlear implantation. In a meta-analysis, Ibrahim et al. [18] reported a detrimental effect of CI surgery on VEMP results. However, they observed that most studies utilized ACS and measured only cVEMPs. Only a few studies measured cVEMPs to $\mathrm{BCV}$ or to both ACS and BCV [1, 6, 10, 35, 43], and even less studies included oVEMP testing [2, $25,45]$. The observed response rates and pathologic asymmetries differ substantially (range 17 to $80 \%$, [18]) between studies and are most likely attributed to the different methods used. In CI patients, absent VEMP responses to ACS could be the result of altered middle and/or inner ear mechanics, i.e., altered sound transmission, rather than loss of otolith function. This poses the risk of misdiagnosis of vestibular dysfunction and thus inaccurate patient counseling, e.g., if a patient is considering implantation on the contralateral side but fears bilateral vestibular loss. Merchant et al. [28] measured VEMPs to ACS and BCV as well as wideband acoustic immittance responses to test for air-bone gaps in CI patients. They reported that response rates to $\mathrm{BCV}$ were higher than to ACS. They also showed that those patients with absent ACS responses but present $\mathrm{BCV}$ responses demonstrated the largest decrease in low-frequency absorbance, i.e., possibly mechanical changes in the middle and/or inner ear induced by the CI leading to air-bone gaps and thus the absence of VEMPs to ACS.

$\mathrm{BCV}$ stimuli can be generated by audiometric BC transducers, e.g. B71 or B81 (Radioear, New Eagle, USA), large vibratory shakers such as the MiniShaker 4810 (Brüel \& Kjær Sound \& Vibration Measurement A/S, Denmark) or tendon hammers. With its high maximum output force, the Mini-Shaker has been used successfully in many studies to measure VEMPs but is rather heavy and bulky and is not approved as a medical device in the EU or the USA. The shaker is originally intended for accelerometer calibration, vibration testing of small objects, and mechanical impedance and mobility measurements. Tendon hammers only allow to assess whether a VEMP response is present or absent. The procedure 
cannot be calibrated with reference to force level, since manual tapping is difficult to control. This makes threshold measurements and comparisons difficult. The relatively new audiometric BC transducer $\mathrm{B} 81$ is a powerful $\mathrm{BC}$ transducer based on the balanced electromagnetic separation transducer (BEST) principle which can produce higher output levels with less harmonic distortion than the B71. Fredén Jansson et al. [22] investigated the electroacoustic performance of the two transducers and found a 7 $\mathrm{dB}$ higher output for the $\mathrm{B} 81$ at $500 \mathrm{~Hz}$ when the transducers were driven by an input voltage of 1 $\mathrm{V}_{\mathrm{RMS}}$. For audiometric applications, the maximum output which can be generated by the transducers is defined as the output which is generated while the harmonic distortion is $<5.5 \%$ and the input voltage is not exceeding $6 \mathrm{~V}_{\mathrm{RMS}}$ (the maximum voltage at which the transducers can be safely operated continuously), whichever occurs first. Harmonic distortion is the limiting factor at the low frequencies. At $500 \mathrm{~Hz}$, a typical frequency used for VEMP measurements, the maximum output by the $\mathrm{B} 81$ is approximately $15 \mathrm{~dB}$ higher compared to the B71, if the distortion levels are to remain below $5.5 \%$. The recording of VEMPs is not intrinsically covered by standards required for audiometric testing. However, audiometric standards were applied in this study because the same instrument (B81) is being used for both audiometry and VEMPs. The lower output by the B71 compared to the B81 could theoretically be (partially) compensated for by increasing drive voltages, if audiometric standards were neglected. However, clinical recording systems using the $\mathrm{B} 71$ or $\mathrm{B} 81$ are calibrated according to the audiometric standard and are limited in drive voltage with respect to the specific transducer [19]. Higher drive voltages exceeding the manufacturer's specifications $\left(6 \mathrm{~V}_{\mathrm{RMS}}\right)$ might be used in research applications. Higher drive voltages without heating of the devices could potentially be possible with intermittent driving as used during VEMP measurements. These limits would have to be defined in a specific data sheet for VEMP applications. However, a standard for VEMP measurements does not exist so far so that audiometric standards were applied in this study.

The B71 was used to elicit VEMPs in previous studies $[21,36]$. Only a few data are published about the use of the B81 for VEMP measurements. Mueller et al. [30] measured oVEMPs in healthy subjects but did not include cVEMPs. In a previous study, we showed that the B81 placed on the mastoid was feasible to elicit cVEMP and oVEMP responses in a group of healthy subjects [13]. The response rates for cVEMPs were 100\%, and for oVEMPs, response rates were $83 \%$ and $92 \%$ (left and right sides) and thus, on average, higher than the response rates to ACS (87\% and $78 \%$ for the left and right sides). To date, there are no studies about the applicability of the B81 on the mastoid to measure VEMPs in patients with (inner) ear pathologies, especially CIs posing the risk of causing an unknown air-bone gap.

The objectives of this exploratory study were to investigate cVEMP and oVEMP response rates to $\mathrm{ACS}$ and $\mathrm{BCV}$ in $\mathrm{CI}$ patients and the applicability of the B81 transducer as an approved medical device placed on the mastoid for BCV stimulation. Therefore, unilateral CI patients were included and the contralateral sides were considered as within-subject control measures.

\section{Methods}

\subsection{Study design and participants}

The study was designed as a prospective comparative experimental study at a single tertiary referral center, including patients who were unilaterally implanted with a CI for at least 8 weeks. The patients' medical charts were reviewed, excluding patients with known vestibular/neurotologic disorders (e.g. vestibulopathy, Menière's disease, vestibular migraine), cochleovestibular schwannoma, cochlear malformations, and fibrosis. Surgical protocols were also reviewed to ensure that the ossicular chain was intact after cochlear implantation. All participants had to be free of active ear infection for the study tests. Otoscopy had been performed in all patients and only patients with an air-bone gap $\leq 10$ $\mathrm{dB}$ at $500 \mathrm{~Hz}$ on the contralateral non-implanted side were included. Screening audiograms were conducted before inclusion to the study.

Written informed consent was obtained from all participants. The study protocol was reviewed and approved by the responsible institutional review board (approval number: 2019-099), and conducted according to the Declaration of Helsinki.

\subsection{VEMP testing}

All VEMP recordings were collected and analyzed using the Eclipse recording platform (Interacoustics A/S, Middelfart, Denmark). After the skin was prepared to provide impedances of $5 \mathrm{k} \Omega$ or less, self-adhesive Ambu Neuroline 720 surface 
electrodes (Ambu A/S, Ballerup, Denmark) were used to record the electromyogram (EMG). EMG signals were recorded in a -20 to $80 \mathrm{~ms}$ time window relative to the onset of the stimulus and were bandpass filtered between $10-1000 \mathrm{~Hz}$. The artifact rejection level was set to $\pm 400 \mu \mathrm{V}$.

The CI was turned off during all VEMP measurements to avoid electrical co-stimulation of vestibular structures due to current spread from the CI. The audio processor was not worn during any VEMP recordings.

For cVEMP measurements, the electrodes were placed over the middle of the sternocleidomastoid muscle ipsilateral to the stimulated side and over the sternum. The patients were sitting on a chair and were asked to turn their head to the shoulder contralateral to the stimulated side for sufficient tonic muscle contraction [33]. The background EMG was calculated from the root mean square (RMS) of the EMG signal averaged over the pre-stimulus window and for each recording frame for this purpose. The signal was constantly monitored and feedback was provided by the examiner, if the tonic muscle contraction was dropping below a minimum of $50 \mu \mathrm{V}$ or exceeding a maximum of $200 \mu \mathrm{V}$. If the patients could not hear the instructions due to insufficient hearing on the contralateral side and with the CI turned off during the measurements, the feedback was given visually by hand signals. The background EMG was also applied to normalize the cVEMP p13-n23 peakto-peak amplitude to the tonic muscle activation in order to reduce the impact of muscle contraction on cVEMP amplitudes.

For oVEMP recordings, the electrodes were placed as bipolar montage on the infra-orbital ridge $1 \mathrm{~cm}$ below the lower eyelid contralateral to the stimulated side and about $2 \mathrm{~cm}$ inferior to the first electrode. The patients lay in supine position and were asked to look up to maintain an upward gaze of $20-30^{\circ}$.

VEMP responses were averaged to at least 200 stimuli for cVEMPs and oVEMPs. The stimuli were short $500 \mathrm{~Hz}$ tone bursts (0-1-0, i.e.: 0 cycles rise/fall time, 1 cycle plateau). For stimulation by BCV, the B81 transducer was fitted with the standard P333 steel spring headband (Radioear, New Eagle, USA) and placed on the ipsilateral mastoid. The stimulus polarity was chosen to produce compressive initial movement, i.e., a force towards the head. For ACS stimulation, ER-3A insert earphones (3M, St. Paul, MS, USA) were used. The maximum stimulus intensity for BCV with the B81 and the Eclipse was 142 $\mathrm{dB}$ peVFL (peak-to-peak equivalent vibratory force levels). For ACS, the maximum stimulus intensity was $100 \mathrm{~dB} \mathrm{nHL}$. The initial stimulus intensity was set at the maximum intensity for all VEMP recordings. To detect the VEMP thresholds, the stimulus intensity was reduced in steps of $5 \mathrm{~dB}$ until no response could be recorded. If no response could be recorded at the maximum intensity, at least two trials were completed to avoid false negative results.

The cVEMP and oVEMP responses were recorded for stimulation by ACS and BCV on both the side implanted with the CI (CI side) and the contralateral, non-implanted side. The contralateral side was used as a within-subject control measurement.

\subsection{VEMP data analysis}

The VEMP data were analyzed in OtoAccess software (Interacoustics A/S, Middelfart, Denmark) by two examiners. When the response was clearly larger than the pre-stimulus waveforms, i.e., the background noise, a VEMP was judged to be present.

The amplitudes were measured as peak to peak, i.e., p13-n23 for cVEMPs, and n10-p15 for oVEMPs. The asymmetry ratio (AR) in \% was calculated from the peak to peak amplitudes by diving the difference between the amplitude of the CI side and the contralateral non-implanted side by the sum of the amplitudes multiplied by 100 . Therefore, a signed AR was used to account for the implanted side (CI) and the contralateral non-implanted side. Positive values of the AR indicate larger responses of the CI side, negative values indicate larger responses of the nonimplanted side, respectively. The amplitude was set to 0 for cVEMPs and $0 \mu \mathrm{V}$ for oVEMPs, if no response could be discerned. Thus, the AR was $100 \%$ for unilateral responses on the CI side only and $-100 \%$ for unilateral responses on the non-implanted side only. If no responses could be recorded bilaterally, the AR was $0 \%$.

\subsection{Statistical analysis}

The cVEMP and oVEMP response rates to ACS and $\mathrm{BCV}$ were calculated for the $\mathrm{CI}$ side and the contralateral side, respectively. Differences between the response rates to $\mathrm{ACS}$ and $\mathrm{BCV}$ were analyzed using the Chi-square test. A confidence level of $95 \%$ or above was considered to be significant $(p<0.05)$.

Descriptive statistics were used to report latency, amplitude, threshold, and asymmetry data. Quantitative data were presented as mean, standard deviation $( \pm)$, and range (minimum and maximum). For the 
calculation of group means, only valid responses were considered. Amplitude values of 0 or $0 \mu \mathrm{V}$ and AR values of $0 \%$ (no response) were not included in the calculation but were displayed in figures by special symbols to show all results, i.e., the total number of included patients.

SPSS statistics (IBM, Armonk, New York, USA) was used for all statistical analyses. If appropriate, qualitative data were presented as graphs using GraphPad Prism 8 (GraphPad Software, San Diego, CA, USA).

\section{Results}

Thirteen Patients, nine females, four males (mean age $44 \pm 12$, range 18 to 60 ) were included in the study. The mean contralateral AC pure tone average at $500,1000,2000$, and $4000 \mathrm{~Hz}$ (4PTA) was $46 \pm 26$ dB HL. Six patients had the CI on the right side, in seven patients the left side was implanted. The demographic data as well as the etiology of hearing loss, implant type, and surgical approach are summarized in Table 1. One patient (ID 13) was re-implanted after device failure, the data in Table 1 apply to the new implant.

The response rates to ACS and BCV for cVEMPs and oVEMPs are illustrated in Fig. 1 for the CI and the contralateral side, respectively. On the contralateral side, cVEMPs were present to ACS in 11 patients $(85 \%)$ and to $\mathrm{BCV}$ in 11 patients $(85 \%)$. On the CI side, cVEMPs to ACS could be obtained in 3 patients (23\%) and to BCV in 10 patients $(77 \%)$. Ocular

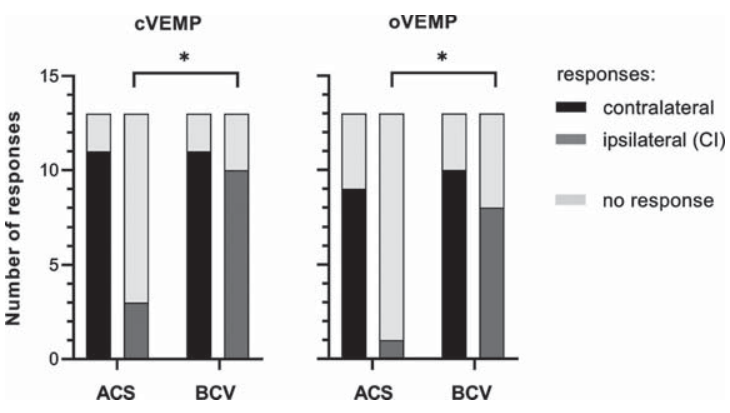

Fig. 1. cVEMP and oVEMP response rates as absolute numbers for the contralateral (control) side (black) and the ipsilateral (CI) side (dark gray) for stimulation by air conduction (ACS) and bone conduction (BCV) with the B81 on the mastoid. Absent responses are illustrated in light gray. Chi-square tests showed that for the ipsilateral (CI) side the oVEMP and cVEMP response rate was higher for $\mathrm{BC}$ stimulation compared to $\mathrm{AC}$ stimulation $(p<0.05$, marked by asterisks).

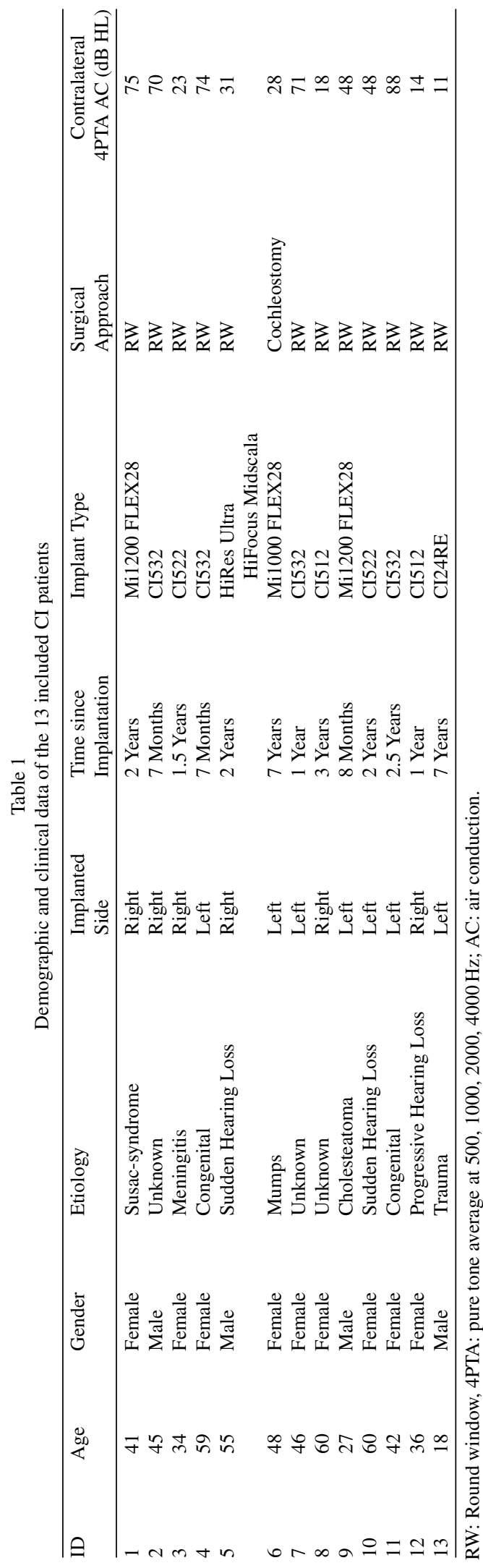


VEMPs on the contralateral side to ACS were measurable in 9 patients $(69 \%)$ and to $\mathrm{BCV}$ in 10 patients (77\%). On the CI side, oVEMPs were present to ACS in 1 patient (8\%) and to $\mathrm{BCV}$ in 8 patients (62\%).

For the CI side, the Chi-square test showed an association between the stimulation mode, i.e., ACS vs. $\mathrm{BCV}$, and the response rate for $\mathrm{cVEMPs}\left(X^{2}(1)=\right.$ 7.54, $p=0.006)$ and for oVEMPs $\left(X^{2}(1)=8.33\right.$, $p=0.004)$. No effect of the stimulation mode on the response rate was observed for the contralateral side.

The amplitudes, asymmetry ratios (ARs), and latencies including means and standard deviations are shown for cVEMPs in Fig. 2. The mean normalized p13-n23 amplitudes to ACS were higher for the contralateral side $(0.9 \pm 0.5, n=11)$ compared to the CI side $(0.3 \pm 0.2, n=3)$. To BCV, the amplitudes were $0.6 \pm 0.4(n=11)$ on the contralateral side and $0.5 \pm 0.3(n=10)$ on the CI side. The AR data show that cVEMPs were absent bilaterally to ACS in 2 patients $(15 \% ; \mathrm{AR}=0 \%)$ and absent unilaterally, on the CI side only (AR $=-100 \%$ ), in 8 patients (62\%). To BCV, bilateral absence was observed in 2 patients $(15 \%)$ and unilateral absence, on the CI side only, in 1 patient $(8 \%)$. The mean AR was therefore lower for ACS $(-88 \pm 23 \%, n=11)$ compared to BCV $(-12 \pm 45 \%, n=10)$. The p13 latencies for the contralateral and the CI side were $14.5 \pm 1.9 \mathrm{~ms}(n=11)$ and $14.1 \pm 1.3 \mathrm{~ms}(n=3)$ for ACS, and $15.1 \pm 2.3 \mathrm{~ms}$ $(n=11)$ and $16.0 \pm 2.0 \mathrm{~ms}(n=10)$ for BCV. The $\mathrm{n} 23$ latencies to ACS were $22.8 \pm 2.4 \mathrm{~ms}(n=11)$ for the contralateral side and $21.2 \pm 1.9 \mathrm{~ms}(n=3)$ for the CI side. To BCV, the latencies were $25.0 \pm 1.5 \mathrm{~ms}$ $(n=11)$ and $24.9 \pm 2.4 \mathrm{~ms}(n=10)$, respectively. The cVEMP thresholds to ACS were $91 \pm 7 \mathrm{~dB}$ nHL

\section{cVEMPs}
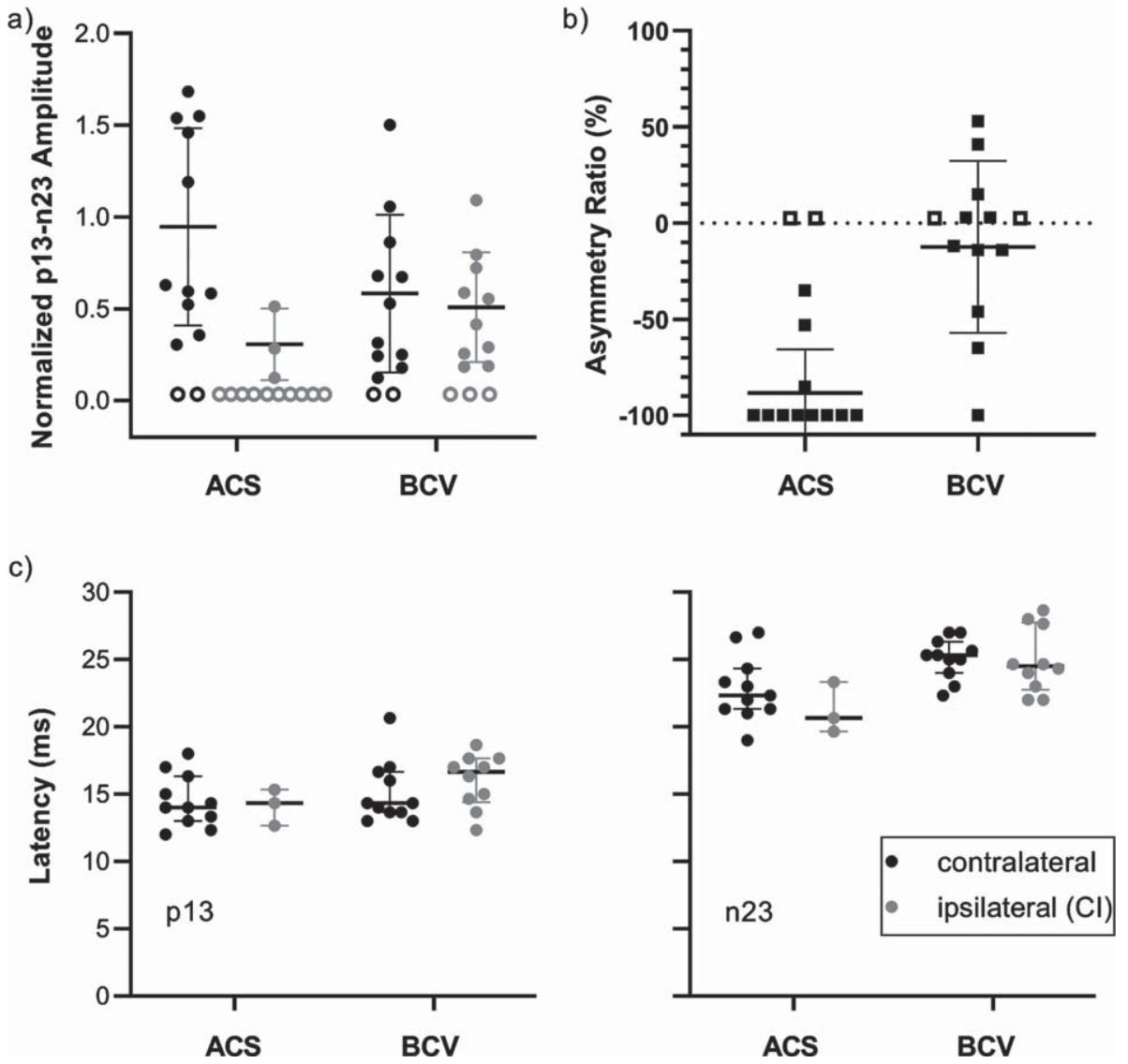

Fig. 2. Overview of cVEMP data for the contralateral (control) side (black) and the ipsilateral (CI) side (dark gray) for stimulation by air conduction (ACS) and bone conduction (BCV) with the B81 on the mastoid. Each data point represents the result of a single patient. Horizontal lines show the means and standard deviations. a) Normalized p13-n23 amplitudes. Patients without responses are represented by empty symbols at 0. b) Signed Asymmetry ratios. Patients with unilateral responses at the contralateral side only are shown at $-100 \%$, with responses on the ipsilateral (CI) side only at $100 \%$, and patients with bilaterally absent responses are represented by empty symbols at $0 \%$. c) p13 and n23 latencies. 
OVEMPS

a)

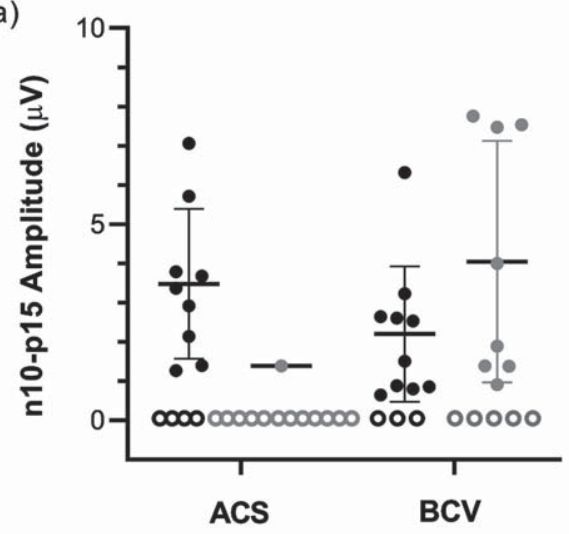

c)

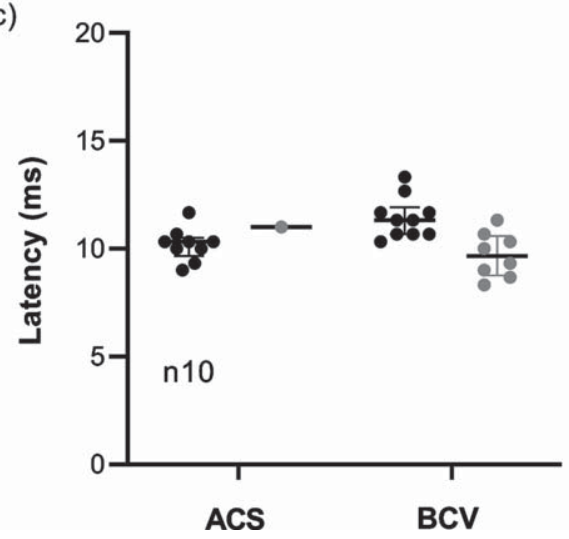

b)
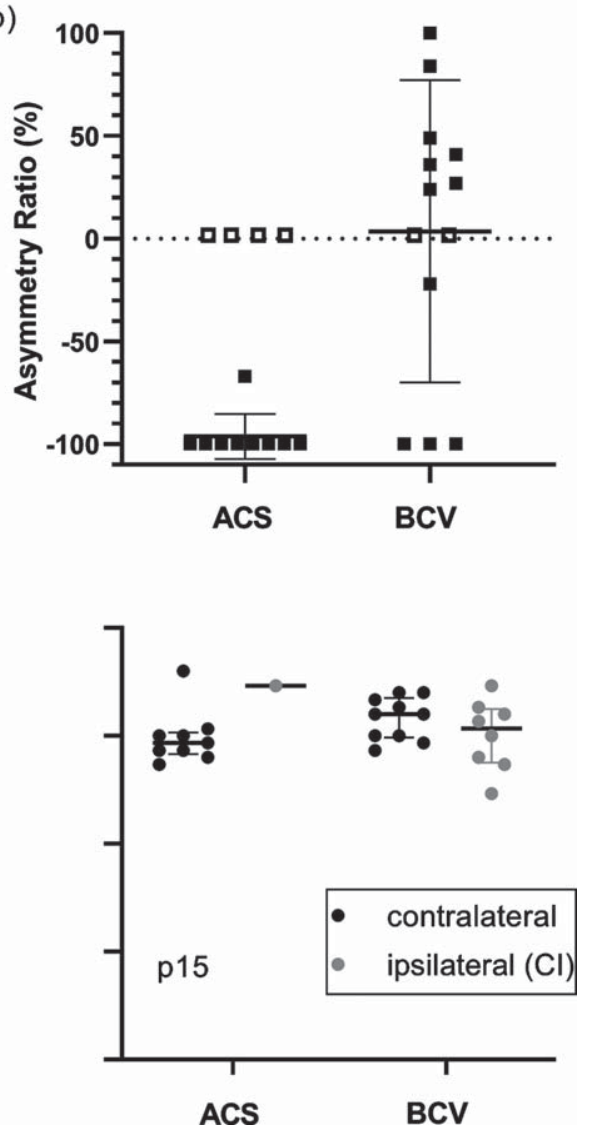

Fig. 3. Overview of oVEMP data for the contralateral (control) side (black) and the ipsilateral (CI) side (dark gray) for stimulation by air conduction (ACS) and bone conduction (BCV) with the B81 on the mastoid. Each data point represents the result of a single patient. Horizontal lines show the means and standard deviations. a) Normalized n10-p15 amplitudes. Patients without responses are represented by empty symbols at $0 \mu \mathrm{V}$. b) Signed Asymmetry ratios. Patients with unilateral responses at the contralateral side only are shown at $-100 \%$, with responses on the ipsilateral (CI) side only at $100 \%$, and patients with bilaterally absent responses are represented by empty symbols at 0\%.c) n10 and p15 latencies.

$(n=11)$ for the contralateral and $95 \pm 5 \mathrm{~dB} \mathrm{nHL}$ $(n=3)$ for the CI side. To BCV, the thresholds were $135 \pm 6 \mathrm{~dB}$ peVFL $(n=11)$ and $136 \pm 4 \mathrm{~dB}$ peVFL $(n=10)$, respectively.

The oVEMP amplitudes, ARs, and latencies with means and standard deviations are illustrated in Fig. 3. Only one patient showed an oVEMP response to ACS on the CI side with an amplitude of $1.4 \mu \mathrm{V}$ which was lower than the mean amplitude on the contralateral side $(3.5 \pm 1.9 \mu \mathrm{V}, n=9)$. To BCV, the mean amplitude was $2.2 \pm 1.7 \mu \mathrm{V}(n=10)$ on the contralateral side and $4.0 \pm 3.1 \mu \mathrm{V}(n=8)$ on the CI side. The AR data show bilaterally absent oVEMPs to ACS in 4 patients $(31 \%)$ and unilateral absence, on the CI side only, in 8 patients $(62 \%)$. For BCV, oVEMPs were absent bilaterally in 2 patients $(15 \%)$ and unilaterally on the CI side in 3 patients (23\%). In 1 patient (8\%), the response was absent unilaterally on the contralateral side. For ACS, the mean AR was lower $(-96 \pm 11 \%, n=9)$ compared to BCV $(4 \pm 74 \%, n=11)$. The mean $n 10$ latencies to ACS were $10.2 \pm 0.8 \mathrm{~ms}(n=9)$ for the contralateral side and $11.0 \mathrm{~ms}(n=1)$ for the CI side. To BCV, the latencies were $11.4 \pm 1.0 \mathrm{~ms}(n=10)$ and $9.7 \pm 1.0 \mathrm{~ms}$ $(n=8)$, respectively. The p15 latencies for the contralateral and the CI side were $14.9 \pm 1.3 \mathrm{~ms}(n=9)$ and $17.3 \mathrm{~ms}(n=1)$ for ACS, and $15.8 \pm 01.0 \mathrm{~ms}$ $(n=10)$ and $15.0 \pm 1.6 \mathrm{~ms}(n=8)$ for BCV. The thresholds for oVEMPs were $95 \pm 6 \mathrm{~dB}$ nHL $(n=9)$ and $95 \mathrm{~dB}$ nHL $(n=1)$ for the contralateral and the $\mathrm{CI}$ side to ACS, and $135 \pm 3 \mathrm{~dB}$ peVFL $(n=10)$ and $132 \pm 5 \mathrm{~dB}$ peVFL $(n=8)$ to $\mathrm{BCV}$. 


\section{Discussion}

The BCV VEMPs in this study were measured using the B81 transducer with audiometric standards applied for operation levels of the transducer. This was done because clinical recording systems using the B81 are calibrated according to the audiometric standard IEC 60645-1 and are limited in drive voltage with respect to the specific transducer [19]. Higher limits for drive voltage with intermittent driving could theoretically be defined by the manufacturer specifically for VEMP measurements. As a standard for VEMP measurements does not exist so far, we applied audiometric standards in our study to operate the transducer within clinically approved limits, i.e., as an approved medical device.

The results from this study show that - for the implanted (CI) side - the stimulation mode had a significant influence on VEMP response rates. For cVEMPs as well as oVEMPs, the response rate increased by $54 \%$ for stimulation by BCV compared to ACS. Response rates also showed tendency to be higher to BCV compared to ACS for oVEMPs on the contralateral non-implanted side but no statistical association could be observed in this case.

One aspect which has to be considered when interpreting cVEMP response rates is that $\mathrm{BCV}$ acts on the saccule and the utricle and utricular afferents project to the SCM (see introduction). Thus, higher response rates for cVEMPs to BCV may be due to an action on the utricle. However, cVEMP response rates for the contralateral side were not higher to BCV $(85 \%)$ compared to ACS (85\%) but the additional activation and influence of utricular afferents should be present in the healthy side as well, if it was a confounding factor. A methodological difference between ACS and BCV in this study was the maximum stimulation level. With respect to the mean cVEMP and oVEMP thresholds to ACS, the maximum AC stimulus was +5 $\mathrm{dB}$ above threshold. For BCV, the maximum BC stimulus was, $+6 \mathrm{~dB}$ above the mean cVEMP threshold and $+10 \mathrm{~dB}$ above the mean oVEMP threshold. However, this difference of $+3 \mathrm{~dB}$ on average between the maximum $\mathrm{BC}$ and $\mathrm{AC}$ stimulus with respect to VEMP thresholds was rather small and was therefore not considered to have a major effect on the results. The latencies and thresholds were not different between $\mathrm{BCV}$ and ACS for both the contralateral and the CI side, indicating that the responses to $\mathrm{BCV}$ on the $\mathrm{CI}$ side were valid VEMP responses, enabling the assessment of vestibular function on the implanted side. In those patients where responses to ACS could be obtained on the CI side, the cVEMP and oVEMP amplitudes to ACS were smaller compared to BCV. Small amplitudes or absent responses, i.e., cVEMP amplitudes of 0 and oVEMP amplitudes of $0 \mu \mathrm{V}$, caused high pathological asymmetries to ACS. To $\mathrm{BCV}$, the asymmetries were lower with mean cVEMP and oVEMP ARs in a normal physiological range $(-12 \pm 45 \%, n=11$ for cVEMPs and $4 \pm 74 \%, n=11$ for oVEMPs). However, the standard deviations and data distributions show that the variability was very high. Thus, normal values for the ARs representing normal otolith function have to be established to define the cut-off criteria for pathologic ARs in CI patients. High variability of ARs was also observed in a prior study in healthy subjects, especially for oVEMPs [14]. The variability is most likely attributed to the stimulation site, since the VEMP to stimulation on the mastoid is sensitive to small changes of transducer placement and results in larger ranges of ARs $[36,38]$. Compared to healthy subjects, the AR variability in the CI patients in this study was found to be even higher. However, several aspects have to be considered for clinical interpretation of ARs in these patients. If otolith function is impaired after implantation, the amplitudes can be reduced, as was shown by negative ARs in our study. However, cochlear implantation can also lead to endolymphatic hydrops [15, 40]. It has been shown, that hydrops can also affect VEMPs leading to enhanced VEMP amplitudes, e.g. in patients with Menière's disease [26, 31, 41, 44, 47] or other inner ear pathologies [12]. Frequency specific VEMP measurements can be used in these cases $[31,41]$.

Although the interpretation of amplitudes and ARs is an important point to consider, results of this study showed very clearly, that the absence of VEMPs due to the use of ineffective stimuli is the major problem. The use of ACS can result in absent VEMPs or small amplitudes and therefore in misinterpretation of vestibular function and inaccurate patient counseling. CI surgery bears the risk of inducing alterations in middle and/or inner ear mechanics and therefore sound conduction [2, 27, 34]. Many patients have also undergone previous middle ear surgeries before treatment with a CI or the etiologies leading to profound hearing loss or deafness, and therefore $\mathrm{CI}$ indication, can also be associated with inhibiting sound transmission themselves. Since the air-bone gap in patients with profound hearing loss cannot be quantified with standard audiometric methods, the use of ACS for VEMP measurement is an ineffective diagnostic method to evaluate otolith function in these patients 
and should be avoided. BCV should be used instead. As the response rate to $\mathrm{BCV}$ was substantially higher than to ACS, it gives more accurate results.

Stimulation by BCV for VEMP testing is not available and therefore not routinely used in all clinics. Large vibratory shakers such as the Mini-Shaker 4810 are effective transducers but not approved as medical devices. They are also costly and have to be calibrated by the examiners themselves. The results from this study showed that the B81 as a standard audiometric bone conduction transducer and approved medical device is a feasible alternative which can be used in any clinical set-up. It can be calibrated to any evoked potential recording system in routine calibration procedures so that only safe stimulation intensities can be used by the examiners. During VEMP testing, the transducer can be coupled to the mastoid using the standard steel spring headbands.

The drawbacks of this exploratory study include the limited sample size with respect to the somehow expected inhomogeneity of CI patients and that it cannot be ruled out that unknown etiologies (or previous middle ear surgeries) had impacted the mechanical sound transduction. The surgical protocols were reviewed for intact ossicular chains but no previous tympanometry or screening questionnaire were performed to rule out confounding middle ear abnormalities at the time of testing. The patients were not asked whether they had a history of dizziness or imbalance, the information was taken from the patients' medical charts to rule out known vestibular disorders. Especially the patient who was re-implanted after device failure would be at even higher risk of surgical otolith damage. However, this patient showed cVEMP and oVEMP responses to $\mathrm{BCV}$ so that he was not excluded from the study. Another drawback is that all patients were recruited after implantation. Preimplantation data was therefore not available and the patients' contralateral sides were tested as within-subject controls. However, the preoperative data would be of great value to determine the effect of cochlear implantation on otolith function, i.e., to examine the changes in (BCV) VEMP responses over time to discern whether the vestibular dysfunction was pre-existing and related to etiology or a direct result of cochlear implantation. Pre- and postoperative VEMP measurements to ACS and BCV should be performed to examine the effect and to evaluate the influence of cochlear implantation on vestibular function.

Regarding the influence of stimulus type, i.e., ACS compared to $\mathrm{BCV}$, on VEMP response rates in $\mathrm{CI}$ patients, only very few data are available in the literature. In a study by Basta et al. [1], cVEMPs were measured pre- and postoperatively in $18 \mathrm{CI}$ patients. To ACS, cVEMPs could not be measured in any patient in their study and to BCV, cVEMPs were present in $33 \%$ of patients. The study by Merchant et al. [28] found cVEMPs to ACS in $41 \%$ and to BCV in $67 \%$ of CI patients (increase of $26 \%$ ). Their study also investigated oVEMPs and recorded responses to ACS in $15 \%$ and to BCV in $52 \%$ of patients (increase of $37 \%$ ). The results reported by Merchant et al. are comparable to the results described in our study. Only for oVEMP recordings, our study shows a considerably lower response rate for ACS. This could be due to the study drawbacks described earlier, i.e., the inclusion/exclusion criteria of our study participants, but also due to the use of different stimuli, recording techniques, and equipment in general, making comparisons difficult. All studies investigating the difference between response rates to $\mathrm{ACS}$ and $\mathrm{BCV}$, including our study, show higher response rates to $\mathrm{BCV}$ and therefore underline the importance of using an effective stimulus for VEMP testing in CI patients.

\section{Conclusion}

ACS is not an effective stimulus to test otolith function by recording VEMPs in patients treated with a cochlear implant (CI). In contrast, BCV was feasible to elicit higher response rates and thus more reliable results. While the use of ACS bears the risk of false negative results, $\mathrm{BCV}$ is an effective stimulus enabling the assessment of utricular and saccular function in CI patients. Absent VEMP responses to ACS must be interpreted with caution and BCV should be implemented as the standard stimulation mode to test these patients. The B81 as a standard audiometric bone conduction transducer and approved medical device has proven a feasible stimulator to provide the $\mathrm{BCV}$ stimulus.

BCV should also be considered as an alternative and effective stimulus in any patient with absent VEMPs to ACS. The B81 as a BCV stimulator should be used in other studies and groups of patients to evaluate its feasibility.

\section{Acknowledgments}

The authors thank Claudia Hahn for support with patient recruiting and scheduling of study visits. 


\section{References}

[1] D. Basta, I. Todt, F. Goepel and A. Ernst, Loss of saccular function after cochlear implantation: the diagnostic impact of intracochlear electrically elicited vestibular evoked myogenic potentials, Audiol Neurootol 13 (2008) 187-192.

[2] X. Chen, X. Chen, F. Zhang and Z. Qin, Influence of cochlear implantation on vestibular function, Acta Otolaryngol. (Stockh.) 136 (2016), 655-659.

[3] C.-H. Choi and J.S. Oghalai, Predicting the effect of postimplant cochlear fibrosis on residual hearing, 205 (2005), 193-200.

[4] R.A. Chole, T.E. Hullar and L.G. Potts, Conductive Component After Cochlear Implantation in Patients With Residual Hearing Conservation 23 (2014), 359-364.

[5] J.G. Colebatch, G.M. Halmagyi and N.F. Skuse, Myogenic potentials generated by a click-evoked vestibulocollic reflex., J Neurol Neurosurg Psychiatry 57 (1994), 190-197.

[6] A. Coordes, D. Basta, R. Götze, S. Scholz, R.O. Seidl, A. Ernst and I. Todt, Sound-Induced Vertigo After Cochlear Implantation:, Otol Neurotol 33 (2012), 335-342.

[7] I.S. Curthoys and V. Vulovic, Vestibular primary afferent responses to sound and vibration in the guinea pig, Exp Brain Res 210 (2011), 347-352.

[8] I.S. Curthoys, V. Vulovic, A.M. Burgess, L. Manzari, L. Sokolic, J. Pogson, M. Robins, L.E. Mezey, S. Goonetilleke, E.D. Cornell and H.G. MacDougall, Neural basis of new clinical vestibular tests: otolithic neural responses to sound and vibration, Clin Exp Pharmacol Physiol 41 (2014), 371-380.

[9] I.S. Curthoys, V. Vulovic, A.M. Burgess, L. Sokolic and S.C. Goonetilleke, The response of guinea pig primary utricular and saccular irregular neurons to bone-conducted vibration (BCV) and air-conducted sound (ACS), Hear Res 331 (2016), 131-143.

[10] B. Devroede, I. Pauwels, S.-D. Le Bon, J. Monstrey and A.-L. Mansbach, Interest of vestibular evaluation in sequentially implanted children: Preliminary results, Eur Ann Otorhinolaryngol Head Neck Dis 133(Suppl 1) (2016), S7-S11.

[11] K.-J. Fredén Jansson, B. Håkansson, S. Reinfeldt, L. Fröhlich and T. Rahne, Vibrotactile Thresholds on the Mastoid and Forehead Position of Deaf Patients Using Radioear B71 and B81:, Ear Hear 38 (2017), 714-723.

[12] L. Fröhlich, I.S. Curthoys, S. Kösling, D. Obrist, T. Rahne and S.K. Plontke, Cervical and Ocular Vestibular-Evoked Myogenic Potentials in Patients With Intracochlear Schwannomas, Front Neurol 11 (2020), 549817.

[13] L. Fröhlich, M. Wilke, S. Plontke and T. Rahne, Influence of bone conduction transducer type and placement on ocular and cervical vestibular evoked myogenic potentials, Sci Rep 11 (2021), 8500.

[14] L. Fröhlich, M. Wilke, S.K. Plontke and T. Rahne, Influence of bone conduction transducer type and placement on ocular and cervical vestibular evoked myogenic potentials, Available Res Sq. PREPRINT (Version 1) (2021).

[15] N.T. Greene, J.K. Mattingly, H.A. Jenkins, D.J. Tollin, J.R. Easter and S.P. Cass, Cochlear Implant Electrode Effect on Sound Energy Transfer Within the Cochlea During Acoustic Stimulation:, Otol Neurotol 36 (2015), 1554-1561.

[16] M.-L. Ho, G. Moonis, C.F. Halpin and H.D. Curtin, Spectrum of Third Window Abnormalities: Semicircular Canal Dehiscence and Beyond, Am J Neuroradiol 38 (2017), 2-9.
[17] A.M. Huber, S.J. Hoon, B. Sharouz, B. Daniel and E. Albrecht, The Influence of a Cochlear Implant Electrode on the Mechanical Function of the Inner Ear, Otol Neurotol 31 (2010), 215-518.

[18] I. Ibrahim, S.D. da Silva, B. Segal and A. Zeitouni, Effect of cochlear implant surgery on vestibular function: metaanalysis study, J Otolaryngol - Head Neck Surg 46 (2017), 44.

[19] IEC 60645-1, Electroacoustics - Audiological equipment Part1: Pure tone audiometers, (2002).

[20] S. Iwasaki, L.A. McGarvie, G.M. Halmagyi, A.M. Burgess, J. Kim, J.G. Colebatch, I.S. Curthoys, Head taps evoke a crossed vestibulo-ocular reflex, Neurology 68 (2007), $1227-1229$.

[21] S. Iwasaki, Y.E. Smulders, A.M. Burgess, L.A. McGarvie, H.G. Macdougall, G.M. Halmagyi and I.S. Curthoys, Ocular vestibular evoked myogenic potentials to bone conducted vibration of the midline forehead at Fz in healthy subjects, Clin Neurophysiol 119 (2008), 2135-2147.

[22] K.-J.F. Jansson, B. Hakansson, L. Johannsen and T. Tengstrand, Electro-acoustic performance of the new bone vibrator Radioear B81: A comparison with the conventional Radioear B71, Int J Audiol 54 (2015), 334-340.

[23] J. Kiefer, F. Böhnke, O. Adunka and W. Arnold, Representation of acoustic signals in the human cochlea in presence of a cochlear implant electrode, Hear Res 221 (2006), 36-43.

[24] K. Kushiro, M. Zakir, Y. Ogawa, H. Sato and Y. Uchino, Saccular and utricular inputs to sternocleidomastoid motoneurons of decerebrate cats, Exp Brain Res 126 (1999), 410-416.

[25] X. Li and S. Gong, The Effect of Cochlear Implantation on Vestibular Evoked Myogenic Potential in Children, The Laryngoscope 130 (2020), E918-E925.

[26] L. Manzari, A.-R. Tedesco, A.M. Burgess and I.S. Curthoys, Ocular and cervical vestibular-evoked myogenic potentials to bone conducted vibration in Ménière's disease during quiescence vs during acute attacks, Clin Neurophysiol 121 (2010), 1092-1101.

[27] J.K. Mattingly, K.M. Uhler and S.P. Cass, Air-Bone Gaps Contribute to Functional Hearing Preservation in Cochlear Implantation, Otol Neurotol 37 (2016), 1255-1262.

[28] G.R. Merchant, K.M. Schulz, J.N. Patterson, D. Fitzpatrick and K.L. Janky, Effect of Cochlear Implantation on Vestibular Evoked Myogenic Potentials and Wideband Acoustic Immittance, Ear Hear 41 (2020), 1111-1124.

[29] S.N. Merchant, H.H. Nakajima, C. Halpin, N. Jr, D.J. Lee, W.P. Innis, H. Curtin and J.J. Rosowski, Clinical Investigation and Mechanism of Air-Bone Gaps in LargeVestibular Aqueduct Syndrome (2008), 15.

[30] A.L. Mueller, L.B. Liebmann, M.R. Petrak, C.M. Bahner, L.M. Weberling, A.D. Weiss and A.J. Matsuoka, Evaluation of the utricular function with the virtual-subject visual vertical system: comparison with ocular vestibularevoked myogenic potentials, Acta Otolaryngol. (Stockh.) 140 (2020), 366-372.

[31] T. Murofushi, H. Ozeki, A. Inoue and A. Sakata, Does Migraine-Associated Vertigo Share a Common Pathophysiology With Meniere's Disease? Study With VestibularEvoked Myogenic Potential, Cephalalgia 29 (2009), 1259-1266.

[32] S.J. O'Leary, Relations between cochlear histopathology and hearing loss in experimental cochlear implantation, Hear Res 298 (2013), 27-35.

[33] T. Rahne, C. Weiser and S. Plontke, NeurofeedbackControlled Comparison of the Head Elevation versus Head 
Rotation and Head-Hand Methods in Eliciting Cervical Vestibular Evoked Myogenic Potentials, Audiol Neurotol 19 (2014), 327-335.

[34] E. Raveh, J. Attias, B. Nageris, L. Kornreich and D. Ulanovski, Pattern of hearing loss following cochlear implantation, Eur Arch Otorhinolaryngol 272 (2015), 2261-2266.

[35] L. Robard, M. Hitier, C. Lebas and S. Moreau, Vestibular function and cochlear implant, Eur. Arch. Oto-RhinoLaryngol. Off. J. Eur. Fed. Oto-Rhino-Laryngol. Soc. EUFOS Affil. Ger. Soc. Oto-Rhino-Laryngol. - Head Neck Surg 272 (2015) 523-530.

[36] S.M. Rosengren, S. Govender and J.G. Colebatch, Ocular and cervical vestibular evoked myogenic potentials produced by air- and bone-conducted stimuli: Comparative properties and effects of age, Clin Neurophysiol 122 (2011), 2282-2289.

[37] S.M. Rosengren, N.P. McAngus Todd and J.G. Colebatch, Vestibular-evoked extraocular potentials produced by stimulation with bone-conducted sound, Clin Neurophysiol 116 (2005) 1938-1948.

[38] S.M. Rosengren, N.P.M. Todd and J.G. Colebatch, Vestibular evoked myogenic potentials evoked by brief interaural head acceleration: properties and possible origin, $J$ Appl Physiol 107 (2009) 841-852.

[39] T. Seo, A. Miyamoto, N. Saka, K. Shimano, T. Nishida, M. Hashimoto and M. Sakagami, Vestibular evoked myogenic potential induced by bone-conducted stimuli in patients with conductive hearing loss, Acta Otolaryngol. (Stockh.) 128 (2008), 639-643.

[40] H. Smeds, H.T. Eastwood, A.J. Hampson, P. Sale, L.J. Campbell, B.D. Arhatari, S. Mansour and S.J. O'Leary, Endolymphatic hydrops is prevalent in the first weeks following cochlear implantation, Hear Res 327 (2015), 48-57.
[41] R.L. Taylor, A.S. Zagami, W.P. Gibson, D.A. Black, S.R. Watson, M.G. Halmagyi and M.S. Welgampola, Vestibular evoked myogenic potentials to sound and vibration: characteristics in vestibular migraine that enable separation from Menière's disease, Cephalalgia 32 (2012), 213-225.

[42] N.P.M. Todd, S.M. Rosengren, S.T. Aw and J.G. Colebatch, Ocular vestibular evoked myogenic potentials (OVEMPs) produced by air- and bone-conducted sound, Clin Neurophysiol 118 (2007), 381-390.

[43] J.H. Wagner, D. Basta, F. Wagner, R.O. Seidl, A. Ernst and I. Todt, Vestibular and taste disorders after bilateral cochlear implantation, Eur Arch Otorhinolaryngol 267 (2010), 1849-1854.

[44] M.-H. Wen, P.-W. Cheng and Y.-H. Young, Augmentation of Ocular Vestibular-Evoked Myogenic Potentials via Bone-Conducted Vibration Stimuli in Ménière Disease, Otolaryngol Neck Surg 146 (2012), 797-803.

[45] X.-D. Xu, X.-T. Zhang, Q. Zhang, J. Hu, Y.-F. Chen and M. $\mathrm{Xu}$, Ocular and cervical vestibular-evoked myogenic potentials in children with cochlear implant, Clin Neurophysiol Off J Int Fed Clin Neurophysiol 126 (2015), 1624-1631.

[46] E.D. Young, C. Fernández and J.M. Goldberg, Responses of Squirrel Monkey Vestibular Neurons to Audio-Frequency Sound and Head Vibration, Acta Otolaryngol. (Stockh.) 84 (1977), 352-360.

[47] Y.-H. Young, C.-C. Wu and C.-H. Wu, Augmentation of Vestibular Evoked Myogenic Potentials: An Indication for Distended Saccular Hydrops:, The Laryngoscope 112 (2002), 509-512.

[48] G. Zhou, D. Poe and Q. Gopen, Clinical Use of Vestibular Evoked Myogenic Potentials in the Evaluation of Patients With Air-Bone Gaps:, Otol Neurotol 33 (2012), 1368-1374. 